\title{
Protocadherins mediate dendritic self-avoidance in the mammalian nervous system
}

\author{
Julie L. Lefebvre ${ }^{1}$, Dimitar Kostadinov ${ }^{1}$, Weisheng V. Chen ${ }^{2}$, Tom Maniatis ${ }^{2}$ \& Joshua R. Sanes ${ }^{1}$
}

\begin{abstract}
Dendritic arborizations of many neurons are patterned by a process called self-avoidance, in which branches arising from a single neuron repel each other ${ }^{1-7}$. By minimizing gaps and overlaps within the arborization, self-avoidance facilitates complete coverage of a neuron's territory by its neurites ${ }^{1-3}$. Remarkably, some neurons that display self-avoidance interact freely with other neurons of the same subtype, implying that they discriminate self from non-self. Here we demonstrate roles for the clustered protocadherins (Pcdhs) in dendritic self-avoidance and self/non-self discrimination. The $P c d h$ locus encodes 58 related cadherin-like transmembrane proteins, at least some of which exhibit isoform-specific homophilic adhesion in heterologous cells and are expressed stochastically and combinatorially in single neurons ${ }^{7-11}$. Deletion of all $22 \mathrm{Pcdh}$ genes in the mouse $\gamma$-subcluster (Pcdhg genes) disrupts self-avoidance of dendrites in retinal starburst amacrine cells (SACs) and cerebellar Purkinje cells. Further genetic analysis of SACs showed that Pcdhg proteins act cell-autonomously during development, and that replacement of the 22 Pcdhg proteins with a single isoform restores self-avoidance. Moreover, expression of the same single isoform in all SACs decreases interactions among dendrites of neighbouring SACs (heteroneuronal interactions). These results suggest that homophilic Pcdhg interactions between sibling neurites (isoneuronal interactions) generate a repulsive signal that leads to self-avoidance. In this model, heteroneuronal interactions are normally permitted because dendrites seldom encounter a matched set of Pcdhg proteins unless they emanate from the same soma. In many respects, our results mirror those reported for Dscam1 (Down syndrome cell adhesion molecule) in Drosophila: this complex gene encodes thousands of recognition molecules that exhibit stochastic expression and isoform-specific interactions, and mediate both self-avoidance and self/non-self discrimination $^{4-7,12-15}$. Thus, although insect Dscam and vertebrate Pcdh proteins share no sequence homology, they seem to underlie similar strategies for endowing neurons with distinct molecular identities and patterning their arborizations.
\end{abstract}

The 58 genes of the mouse $P c d h$ locus are tandemly arranged in $\alpha-, \beta$ and $\gamma$-subclusters, called Pcdha, Pcdhb and Pcdhg, which encode 14, 22 and 22 cadherin-like proteins, respectively ${ }^{8}$ (Fig. 1a). In the Pcdha and Pcdhg subclusters, single variable exons encoding extracellular, transmembrane and juxtamembrane domains are spliced to three constant exons, generating proteins with unique extracellular but common intracellular domains ${ }^{8}$. The complexity of this locus is reminiscent of that of Dscam1, which mediates self-avoidance in Drosophila ${ }^{4-7,15}$. Moreover, Pcdh genes, like Dscam1, exhibit stochastic expression, and both Pcdhg and Dscam proteins exhibit isoform-specific homotypic recognition ${ }^{13,14}$. In contrast, the two vertebrate Dscams are not complex genes, so although they mediate both repulsive and attractive interactions among neurons ${ }^{16-19}$, they are unlikely to underlie self/non-self discrimination. We therefore investigated roles of $P c d h$ genes in these processes.

Previous studies of mouse mutants lacking all 22 Pcdhg genes revealed that they are required for survival of multiple neuronal types $^{20-23}$. To seek roles of Pcdhgs in self-avoidance, we focused on a retinal interneuron, the SAC, which expresses Pcdhg genes ${ }^{22}$ and exhibits marked dendritic self-avoidance ${ }^{24}$. Radially symmetric SAC dendritic arborizations are confined to narrow planes within the inner plexiform (synaptic) layer; SACs have no axons. Dendrites of a single SAC seldom cross one another, yet dendrites of neighbouring SACs cross freely (Fig. 1b, c; Supplementary Fig. 1) and even form synapses with each other ${ }^{24,25}$, suggesting that they can distinguish 'self from 'non-self.

We used a conditional mutant $\left(P c d h g^{f c o n 3}\right)^{22}$ to bypass the neonatal lethality of constitutive Pcdhg mutants and employed Cre drivers that delete Pcdhg genes from all or subsets of retinal cells. We visualized individual neurons by infection with recombinant adeno-associated virus (rAAV) expressing a fluorescent protein (XFP; Fig. 1d, e), biolistic delivery of DNA encoding XFP, or intracellular injection of a fluorescent dye. We identified SACs, the sole cholinergic neurons in retina, with antibodies to choline acetytransferase (ChAT), which also demonstrated the association of XFP-positive SAC dendrites with dendrites from other (XFP-negative) SACs (Supplementary Figs 1 and 2).

SAC morphology was profoundly altered in Pcdhg mutant retinas (Pcdhg ${ }^{f c o n 3 / f c o n} 3$; retina-cre, called Pcdhg ${ }^{r k o / r k o}$ here; see Methods for genotypes). Dendrites arising from a single SAC frequently crossed each other and sometimes formed loose bundles (Fig. 1f-i and Supplementary Fig. 1). Crossing frequency was increased several-fold in both proximal and distal regions of the arborization (Fig. 1j). These defects were highly specific, in that the diameter of SAC arborizations, the number of dendritic termini, the laminar targeting of SAC dendrites, and the mosaic arrangement of SAC bodies were all unaffected in $P c d h g^{r k o / r k o}$ mutants (Fig. 1k, 1 and Supplementary Figs 1 and 2). Thus, Pcdhgs are dispensable for many aspects of SAC morphogenesis but are required for their self-avoidance.

In the absence of $P c d h g$ genes, neurons of many types die in elevated numbers during the period of naturally occurring cell death ${ }^{20-23}$. Although SACs are largely spared in Pcdhg mutants ${ }^{22}$, their dendritic defects might be secondary to loss of other neurites with which they ordinarily interact. To test this possibility, we blocked apoptosis by deleting the Bax gene, which is required for naturally occurring and Pcdhg-dependent neuronal death ${ }^{22,23,26}$. SAC morphology was normal in $\mathrm{Bax}^{-{ }_{-}^{-}}$mice, but self-avoidance defects persisted in $\mathrm{Bax}^{-/-}$; Pcdhg ${ }^{r k o / r k o}$ double mutants (Supplementary Fig. 3).

We next asked whether Pcdhgs are required for the development of SAC arborizations, or only for their maintenance. In wild-type neonates, SACs extended dendrites that branched profusely and contacted each other (Fig. 2a-c). By postnatal day (P)12, however, excess neurites and isoneuronal contacts were eliminated, resulting in a radial arborization with evenly spaced branches (Fig. $2 \mathrm{~d}$, and see ref. 24). Thus, self-avoidance arises rapidly following a short period of isoneuronal 'sampling'. In Pcdhg ${ }^{r k o / r k o}$ mice, SACs were clearly aberrant by $\mathrm{P}$, exhibiting excessive crossing and tangling of neurites (Fig. 2e-g). Excess branches were subsequently eliminated, but 


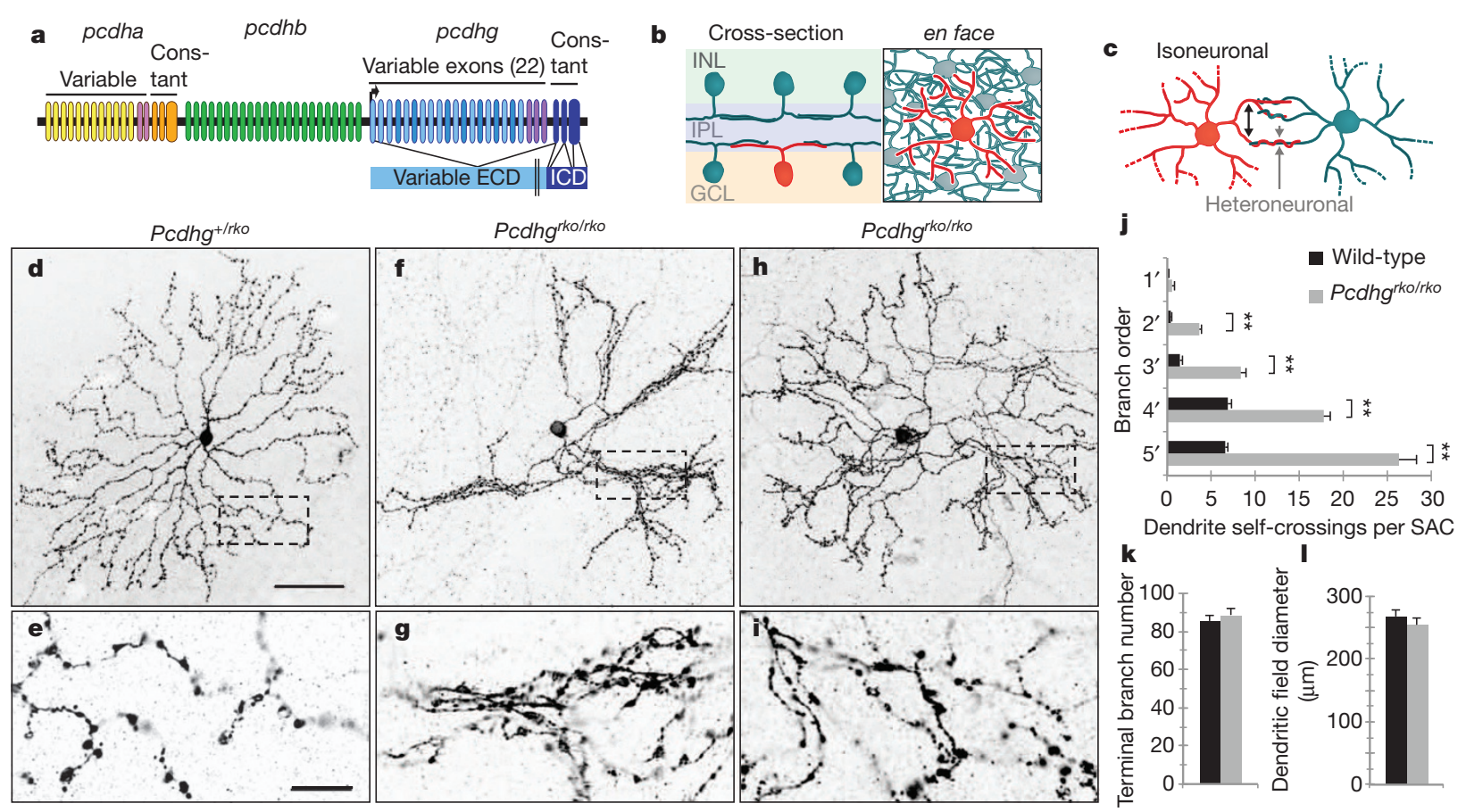

Figure $1 \mid$ Pcdhgs are required for self-avoidance of SAC dendrites. a, $P c d h$ locus comprises $P c d h a, P c d h b$ and $P c d h g$ subclusters. Pcdha and $P c d h g$ isoforms are assembled by splicing of one variable exon encoding extracellular (ECD) and transmembrane domains to three constant exons encoding the intracellular domain (ICD). b, SACs are present in both the inner nuclear layer (INL) and the ganglion cell layer (GCL) and extend dendrites that form radially symmetrical arborizations confined to thin sublaminae in the inner plexiform layer (IPL). c, SAC dendrites avoid isoneuronal dendrites (red) but interact heteroneuronally with other SACs (blue), forming synapses on their dendrites. d-i, Morphology of a single SAC, labelled with membrane-Cherry, in the GCL in control and Pcdhg

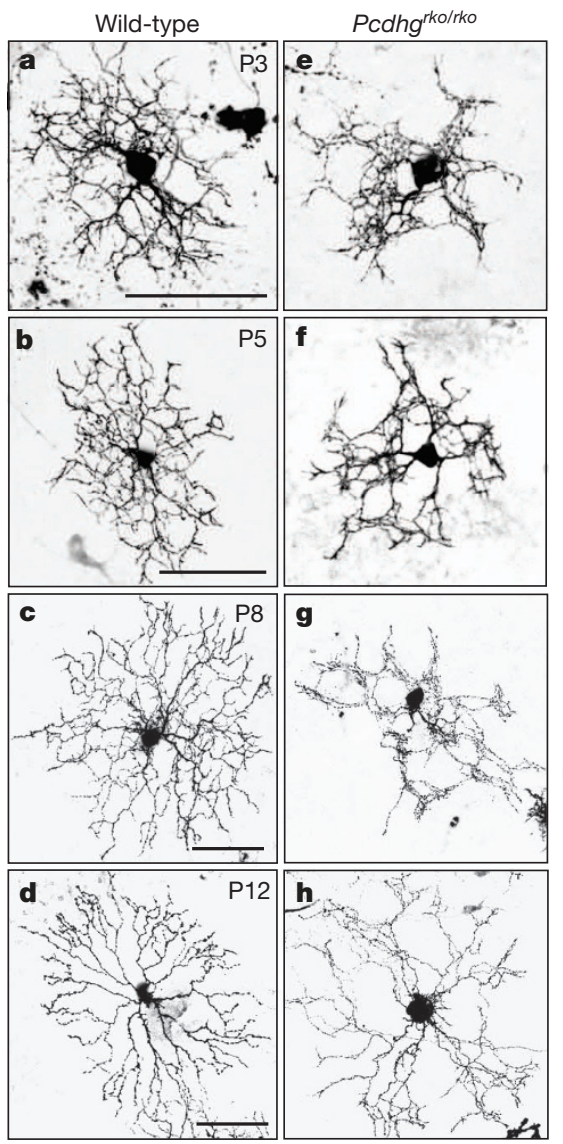

mutant retinas. Wild-type SAC dendrites self-avoid. In Pcdhg mutants, selfavoidance defects include self-crossing and bundling of dendrites. Crossings are detected at $0.2 \mu \mathrm{m} x-y$ resolution in single $0.8-\mu \mathrm{m}$ optical sections $(\mathbf{e}, \mathbf{g}, \mathbf{i}$ show magnified views of boxed areas in $\mathbf{d}, \mathbf{f}, \mathbf{h}$ ). Images with $0.2 \mu \mathrm{m} z$ resolution are shown in Supplementary Fig. 1. j, SAC dendritic self-crossings in first-fifth order branches per SAC. Graph underestimates difference between genotypes because the most severely affected mutant SACs could not be scored. $* * P<0.01$.

$\mathbf{k}, \mathbf{l}$, Number of terminal branches $(\mathbf{k})$ and dendritic field diameter $(\mathbf{l})$ do not differ between wild-type and mutant SACs. Panels $\mathbf{j}-\mathbf{l}$ show means \pm s.e.m.; $n=8$ cells from 5-6 animals per genotype. Scale bars, $50 \mu \mathrm{m}(\mathbf{d}, \mathbf{f}, \mathbf{h})$ and $10 \mu \mathrm{m}(\mathbf{e}, \mathbf{g}, \mathbf{i})$.

whereas most crossing branches were eliminated in controls, many persisted in mutants (Fig. 2h). Thus, Pcdhgs may lead to self-avoidance by mediating repulsive interactions that bias the rearrangement process to selectively eliminate contacts among isoneuronal branches.

To initiate analysis of the mechanism by which Pcdhgs mediate selfavoidance, we next asked whether they act cell-autonomously. We selectively removed Pcdhg genes from SACs using a ChAT-Cre line. In this case, $P c d h g$-negative SACs were surrounded by $P c d h g$-positive neurons of other types. We also deleted $P c d h g$ genes from individual SACs using a transgenic line that expressed tamoxifen-activated Cre recombinase in SACs; we activated Cre with a low dose of tamoxifen and introduced a Cre-dependent reporter to mark mutant SACs. In this case, Pcdhg-negative SACs were surrounded by Pcdhg-positive SACs. In both cases, SACs lacking Pcdhg genes exhibited striking self-avoidance defects (Supplementary Fig. 4). To test whether Pcdhgs can act in completely isolated SACs, we used fluorescenceactivated cell sorting to purify SACs from a transgenic line in which

Figure $2 \mid$ Pcdhgs pattern developing SAC dendrites in a cell-autonomous manner. a-h, SACs in developing wild-type and $P c d h g$ mutant retinas. Wildtype SACs extend fine, exuberant branches (P3, P5) that make transient intradendritic contacts (P5, P8); by P12, excess branches and isoneuronal contacts are eliminated. Dendrites of mutant SACs display excessive selfcrossing and bundling by $\mathrm{P} 3$; by P12, excess branches are eliminated, but crossing dendrites remain. $\mathbf{i}, \mathbf{j}$, Cultured Pcdhg mutant SACs exhibit loss of symmetric growth and uneven distribution of neurites. DIV, days in vitro. k, Histogram of fractal dimensions ( $D_{\mathrm{f}}$ metric for space-filling) for 47 wild-type (black) and 47 mutant (grey) SACs. Wild-type SAC in $\mathbf{i}$ has $D_{\mathrm{f}}$ of 1.61 and mutant SAC in $\mathbf{j}$ has $D_{\mathrm{f}}$ of 1.53. $\mathbf{1}$, Mean $D_{\mathrm{f}}$ for cultured SACs ( $n=47$ cells), SACs in vivo at P5 $(n=6)$ and adult $(n=9)$. $* * * P<0.001$. Error bars, s.e.m. Scale bars, $50 \mu \mathrm{m}(\mathbf{a}-\mathbf{d}$, also apply respectively to $\mathbf{e}-\mathbf{h})$ and $20 \mu \mathrm{m}(\mathbf{i}, \mathbf{j})$. 
they are selectively labelled by an orange fluorescent protein (Thy1OFP3) and cultured them at low density. Isolated SACs extended dendrites that formed radial, web-like arborizations (Fig. 2i), reminiscent of those observed at $\sim \mathrm{P} 5$ in vivo (Fig. 2b). In contrast, SACs from $P c d h g^{r k o / r k o}$; Thy1-OFP3 mice exhibited less symmetrical and unevenly spaced arborizations, reminiscent of those observed in $P c d h g^{r k o / r k o}$ retinas at P5 (Fig. $2 \mathrm{j}$ and Supplementary Fig. 5). Analysis of the space-filling capacity of dendritic arborizations ${ }^{2,27}$ (see Methods) revealed that defects in vitro were similar in magnitude to those in vivo (Fig. 2k, l). Thus, Pcdhgs do not depend on intercellular interactions to promote self-avoidance.

We next assessed the requirement for isoform diversity in Pcdhgdependent self-avoidance. We used RT-PCR (PCR with reverse transcription) to survey expression of Pcdhg isoforms in whole retina, in amacrines generally and in SACs specifically. All 22 Pcdhg variants were expressed in each preparation, with no indication of decreased diversity in purified subpopulations (Supplementary Fig. 6). We then analysed a targeted mouse mutant, $P c d h g^{\text {tcko }}$, in which three

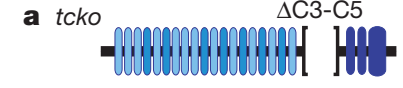

$\Delta$ PcdhgC3-C4-C5 Isoform diversity $=19$

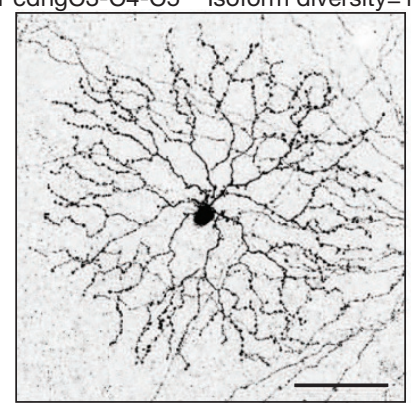

c tako $\Delta \mathrm{A} 1-\mathrm{A} 3$

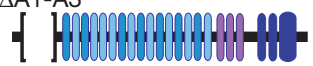

$\triangle \mathrm{PcdhgA} 1-\mathrm{A} 2-\mathrm{A} 3$ Isoform diversity $=19$

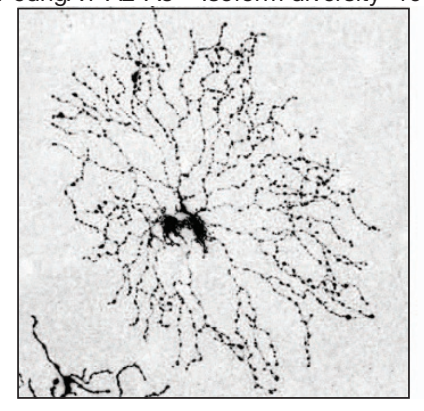

e

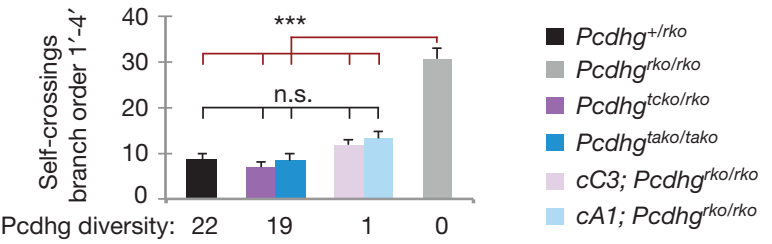

Figure 3 No single Pcdhg isoform is necessary and any isoform is sufficient for dendrite self-avoidance. a, SACs lacking Pcdhgc3-c5

(pcdhg ${ }^{\text {tcko/fcon } 3}$;retina-cre) exhibit self-avoidance. $\mathbf{b}$, Replacement of all 22 Pcdhgs by the PcdhgC3 isoform, using the RC::cC3-cherry transgene, rescues SAC dendrite self-avoidance. c, SACs lacking Pcdhga1-a3 ( $p c d h g^{\text {tako/tako }}$ ) exhibit self-avoidance. d, Replacement of all 22 Pcdhgs by the PcdhgA1 isoform, using the RC::cA1-cherry transgene, rescues SAC dendrite selfavoidance. e, Compared to mutants lacking all 22 isoforms, self-crossings of SACs in retinas expressing 19 or 1 isoforms are restored to control levels.

$* * * P<0.001 ;$ n.s., not significant. Data are mean \pm s.e.m., from 7 SACs from pcdhg ${ }^{\text {tcko/fcon } 3}$; retina-cre retinas, 3 SACs from $p c d h g^{\text {tako/tako }}$, and 9 from remaining genotypes. Scale bar in a, $50 \mu \mathrm{m}$; applies to $\mathbf{b}-\mathbf{d}$ also. contiguous Pcdhg variable exons, C3-C5, had been deleted. Expression of the remaining $19 P c d h g$ isoforms is unperturbed in this allele ${ }^{28}$. Because Pcdhg ${ }^{\text {tcko }}$ homozygous mice die at birth ${ }^{28}$, we generated transheterozygous animals (Pcdhg ${ }^{\text {tcko/fcon } 3}$; retina-cre) so that only retina lacks both copies of Pcdhgc3-c5. In these retinas, neuronal death was as prevalent as in those of $P c d h g^{r k o / r k o}$ mice $^{22,28}$, yet SACs exhibited normal self-avoidance (Fig. 3a, e).

In a complementary approach, we generated a line in which the single PcdhgC3 isoform, fused to a fluorescent protein (mCherry), could be expressed in any cell in a Cre-dependent manner (ROSA26-CAG::lox-Stop-lox-Pcdhgc3-mCherry or cC3-mCherry). Thus, in $c C 3-m C h e r r y ; P c d h g^{r k o / r k o}$ mice, Cre both deletes all 22
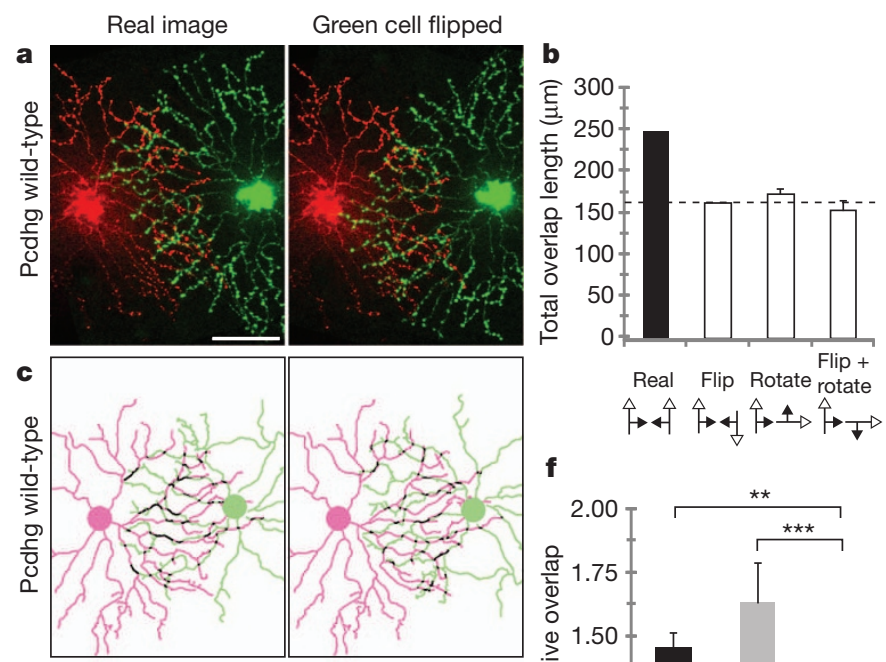

Real Flip Rotate rotate

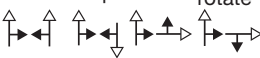

$\mathbf{f}$
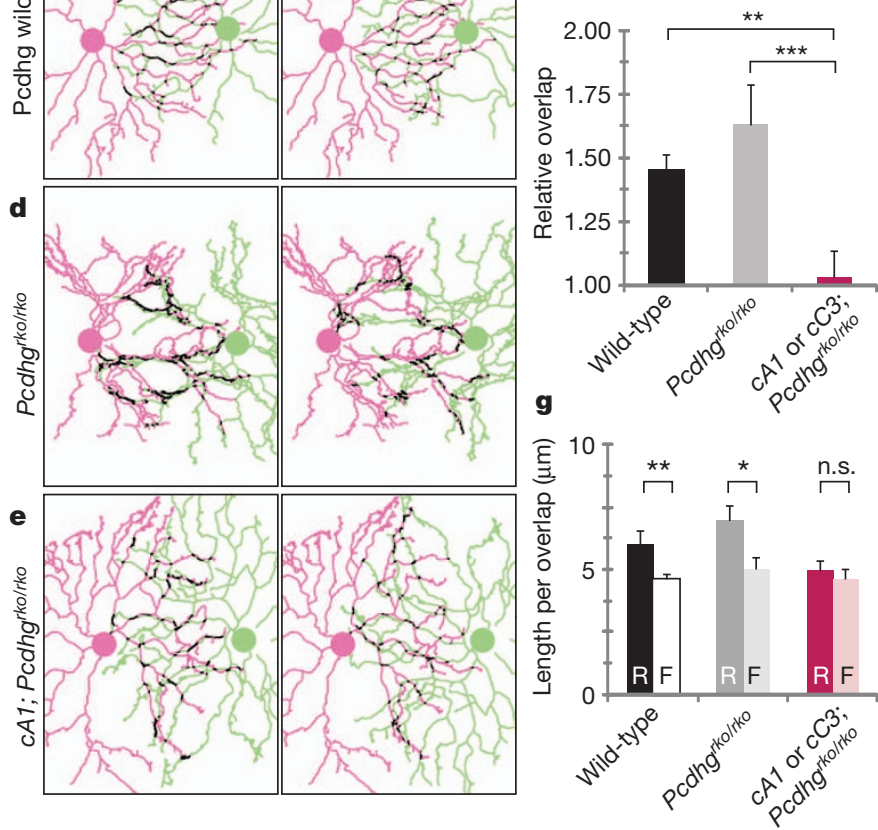

Figure 4 Reducing Pcdhg diversity disrupts heteroneuronal SAC interactions. a, Two nearby SACs from a wild-type mouse injected with contrasting fluorescent dyes. Right panel shows image of the green SAC flipped vertically. $\mathbf{b}$, Overlap between red and green cells in $\mathbf{a}$. First two bars are derived from the two panels in a. The green cell was rotated in $45^{\circ}$ steps or flipped and then rotated (manipulations indicated by symbols beneath graph); third and fourth bars show mean overlap \pm s.e.m. derived from these images $(n=7)$. All inversions and rotations decrease overlap, indicating that overlap in the real image is non-random. c-e, Tracings of SAC pairs, and versions flipped as in a, from wild-type (c), $P c d h g^{r k o / r k o}$ (d) and $c A 1 ; P c d h g^{r k o / r k o}$ (e) mice. Overlap shown in black. f, Overlap between neighbouring cells, expressed as ratio between overlap measured in real and flipped images. Bars show mean \pm s.e.m. for 11,9 and 8 pairs from wild-type, $P c d h g^{r k o / r k o}$ and single isoform-expressing $\left(c A 1 ; P c d h g^{r k o / r k o}\right.$ and $\left.c C 3 ; P c d h g^{r k o / r k o}\right)$ animals. Expression of a single isoform in neighbouring SACs decreases their interaction. g, Mean length of overlapping segments between SAC pairs. R, real image; F, flipped image. $* P=0.05 ; * *<0.05 ; * * * P<0.01$. Error bars, s.e.m.; $n$ as in $\mathbf{f}$. Scale bar in a, $50 \mu \mathrm{m}$; applies to $\mathbf{c}-\mathbf{e}$ also. 
endogenous Pcdhg genes and activates the single PcdhgC3-mCherry isoform throughout the retina. Analysis of mCherry fluorescence confirmed Cre-dependent expression of the transgene in all retinal cells and appropriate localization of the fusion protein to cell membranes and synaptic layers (Supplementary Fig. 7). Expression of Pcdhgc3 alone rescued self-avoidance defects of Pcdhg mutants (Fig. 3b, e).

To test the possibility that only some isoforms are dispensable for self-avoidance, we analysed a second set of isoforms. We generated $P c d h g^{\text {tako }}$, which lacks the Pcdhgal-a3 variable exons ${ }^{28}$, and a line that expresses Pcdhga1-mCherry in a Cre-dependent manner (cA1$m$ Cherry). Results were similar to those for the C3-C5 group: selfavoidance persisted in the absence of PcdhgA1-A3 and was rescued by replacement of all Pcdhg isoforms with PcdhgA1 alone (Fig. 3c-e and Supplementary Fig. 7). From these results, we conclude that no single Pcdhg isoform is necessary but any single isoform is sufficient for dendritic self-avoidance.

Although Pcdhg isoform diversity is not required for isoneuronal self-avoidance, it may be required to ensure that dendrites of adjacent SACs do not avoid each other, which would prevent them from interacting. The ability to generate a SAC population expressing a single Pcdhg isoform (Pcdhgal or Pcdhgc3) enabled us to test this idea. We injected closely spaced pairs of SACs with different fluorophores (Fig. 4a) and measured the extent to which their dendrites overlapped. To determine whether this method reliably revealed interactions among SACs, we rotated, flipped or rotated and flipped the image of one of the cells, and recalculated overlap. Only the real image showed an overlap greater than that of the manipulated images (Fig. 4b). We then measured overlap for pairs of SACs from wild-type, mutant and single isoform-expressing mice, normalizing for intercellular distance by comparing overlap to the value calculated from the flipped image (Fig. 4c-e and Supplementary Fig. 8). Overlap was equivalent in wild-type and mutant retina, but significantly decreased in retinas expressing a single isoform (Fig. 4f); values for Pcdhgal and Pcdhgc3 were similar (1.01 and 1.08). Likewise, the mean length of overlapping segments was greater than expected for random overlap in wild-type and mutant but not in single isoform-expressing pairs (Fig. $4 \mathrm{~g}$ ). Thus, when all SACs express the same Pcdhg isoform, heteroneuronal dendrites avoid each other, just as isoneuronal dendrites do in control SACs. We conclude that isoform diversity enables SACs to distinguish isoneuronal from heteroneuronal dendrites.

Finally, we asked whether Pcdhgs mediate self-avoidance in areas other than retina. We examined cerebellar Purkinje cells, which have elaborate, planar dendritic arborizations known to exhibit selfavoidance $^{3}$ (Fig. 5a-c). Importantly, stochastic and combinatorial expression, which underlies the ability of Drosophila Dscam1 to mediate self-avoidance ${ }^{4-6,12,14,15,29}$, has been documented for Pcdhg genes in Purkinje cells ${ }^{10}$. We selectively deleted Pcdhg genes from Purkinje neurons using an L7-cre transgene, marked cells with a vector that expresses fluorescent proteins in a Cre-dependent manner, and examined them at P15, P21 and at P35, after arborizations have matured $^{30}$. Deletion of $P c d h g$ genes from Purkinje cells had no detectable effect on their survival, shape, size or branching pattern (Fig. 5d, e, h, i and Supplementary Fig. 9), but their arborizations were disorganized and dendrites often crossed over each other (Fig. 5f, g). Use of a Cre-dependent reporter revealed that deletion remained incomplete at P8, at which time Purkinje dendrite growth was already advanced (Supplementary Fig. 9). It is therefore possible that earlier deletion of Pcdhg genes would lead to a more dramatic effect. Nonetheless, these results demonstrate a role for Pcdhg genes in Purkinje cell self-avoidance.

In summary, although vertebrate $P c d h$ genes and Drosophila Dscam1 are structurally unrelated, they have remarkable parallels: both encode numerous isoforms from a single locus, the isoforms are expressed stochastically and combinatorially, and the encoded proteins interact homophilically $7,8,10-14$. We have now shown that in mammalian neurons, Pcdhgs, like Dscam1 (refs 4-6, 12), promote self-avoidance during development by a cell-autonomous mechanism. In addition, for both Dscam 1 and Pcdhg genes, diversity appears to
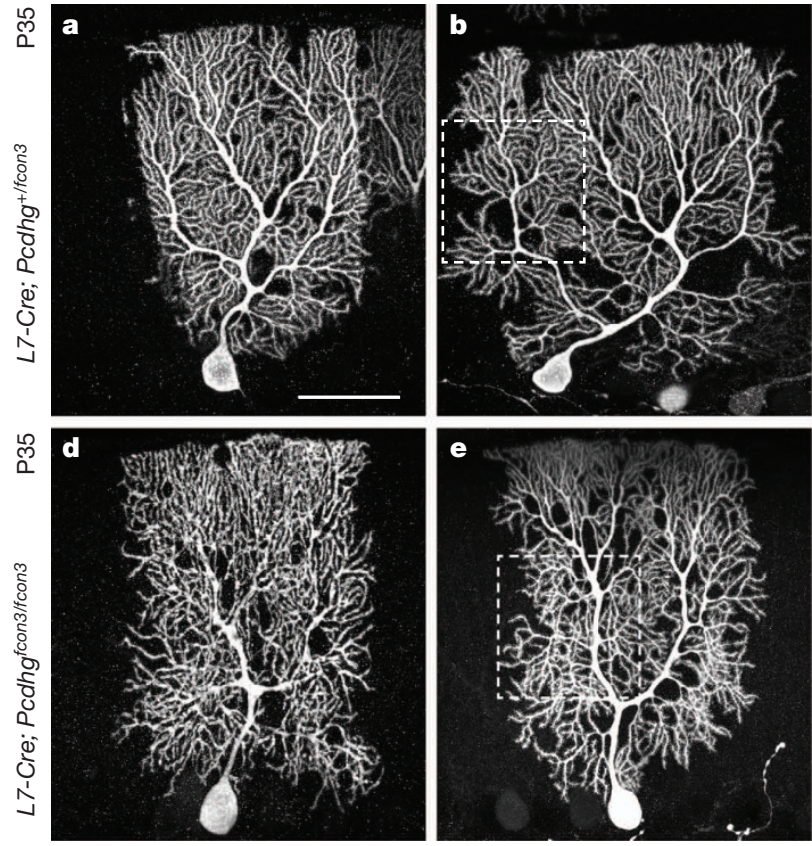

Figure $5 \mid$ Purkinje cell dendrite self-avoidance requires Pcdhgs. a-c, Control Purkinje cells (a, b) labelled with Cre-dependent AAV-XFP in L7cre transgenic mouse. Self-avoidance is clear in high-magnification view in $\mathbf{c}$ (shows area boxed in $\mathbf{b}$ ). $\mathbf{d}-\mathbf{f}$, Purkinje cells lacking Pcdhgs and labelled as in a-c have disorganized arborizations marked by frequent self-crossing defects. Panel $\mathbf{f}$ shows area boxed in e. $\mathbf{g}$, Self-crossings detected in single confocal
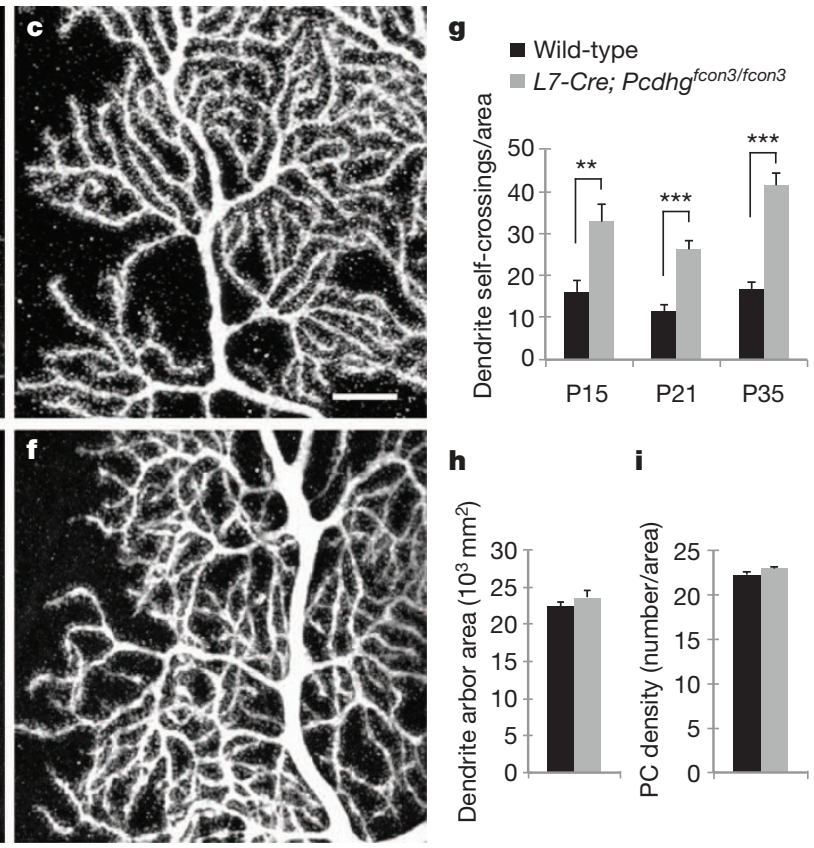

$z$-sections of $7,225 \mu \mathrm{m}^{2}$ unit area from controls and mutants. $* * P<0.01$; $* * * P<0.001 ; n=8,15$ and 15 cells at P15, P21 and P35 respectively from $\geq 3$ mice per genotype. $\mathbf{h}, \mathbf{i}$, Area of dendritic arborizations $(n=20$ cells $)$ and cell density ( $>40$ regions) do not differ between control and mutant Purkinje cells. Data show mean \pm s.e.m. Scale bars, $50 \mu \mathrm{m}(\mathbf{a}, \mathbf{b}, \mathbf{d}, \mathbf{e})$ and $10 \mu \mathrm{m}(\mathbf{c}, \mathbf{f})$. 
underlie self/non-self discrimination, presumably because neighbouring neurons are unlikely to express the same isoforms and are therefore free to interact $t^{7,12,14,15,29}$. Thus, two phyla appear to have recruited different molecules to mediate similar, complex strategies for selfrecognition during formation of neuronal arborizations. These parallels raise the question of why vertebrate and invertebrate nervous systems have invested heavily in mechanisms that promote self-avoidance. In principle, self-avoidance allows neurons to cover their receptive or projective fields maximally while retaining the ability to overlap those of neighbouring neurons ${ }^{1-3}$. However, to our knowledge, the effect of perturbing self-avoidance on circuit function has yet to be assessed in any system. We can now address this issue by electrophysiological analysis of SACs, Purkinje cells, and their synaptic targets in Pcdhg mutant mice.

\section{METHODS SUMMARY}

Transgenic, knockout and knock-in mouse lines used for this study, as well as methods for marking cells are described in Methods. Identity of labelled SACs was confirmed by immunolabelling retinas for the cholinergic neuron-specific marker. Dendrite self-crossings were quantified by number of branch overlaps detected in single confocal planes.

Full Methods and any associated references are available in the online version of the paper.

\section{Received 19 December 2011; accepted 7 June 2012.}

Published online 29 July 2012.

1. Kramer, A. P. \& Kuwada, J. Y. Formation of the receptive fields of leech mechanosensory neurons during embryonic development. J. Neurosci. 3, 2474-2486 (1983).

2. Montague, P. R. \& Friedlander, M. J. Expression of an intrinsic growth strategy by mammalian retinal neurons. Proc. Natl Acad. Sci. USA 86, 7223-7227 (1989)

3. Grueber, W. B. \& Sagasti, A. Self-avoidance and tiling: mechanisms of dendrite and axon spacing. Cold Spring Harb. Perspect. Biol. 2, a001750 (2010).

4. Matthews, B. J. et al. Dendrite self-avoidance is controlled by Dscam. Cell 129, 593-604 (2007)

5. Soba, P. et al. Drosophila sensory neurons require Dscam for dendritic self avoidance and proper dendritic field organization. Neuron 54, 403-416 (2007).

6. Hughes, M. E. et al. Homophilic Dscam interactions control complex dendrite morphogenesis. Neuron 54, 417-427 (2007)

7. Zipursky, S. L. \& Sanes, J. R. Chemoaffinity revisited: dscams, protocadherins, and neural circuit assembly. Cell 143, 343-353 (2010).

8. Wu, Q. \& Maniatis, T. A striking organization of a large family of human neural cadherin-like cell adhesion genes. Cell 97, 779-790 (1999).

9. Kohmura, N. et al. Diversity revealed by a novel family of cadherins expressed in neurons at a synaptic complex. Neuron 20,1137-1151 (1998).

10. Kaneko, R. etal. Allelic gene regulation of $P c d h-\alpha$ and $P c d h-\gamma$ clusters involving both monoallelic and biallelic expression in single Purkinje cells. J. Biol. Chem. 281, 30551-30560 (2006)

11. Schreiner, D. \& Weiner, J. A. Combinatorial homophilic interaction between $\gamma$-protocadherin multimers greatly expands the molecular diversity of cell adhesion. Proc. Natl Acad. Sci. USA 107, 14893-14898 (2010).

12. Zhan, X. L. et al. Analysis of Dscam diversity in regulating axon guidance in Drosophila mushroom bodies. Neuron 43, 673-686 (2004).

13. Wojtowicz, W. M., Flanagan, J. J., Millard, S. S., Zipursky, S. L. \& Clemens, J. C. Alternative splicing of Drosophila Dscam generates axon guidance receptors that exhibit isoform-specific homophilic binding. Cell 118, 619-633 (2004).
14. Neves, G., Zucker, J., Daly, M. \& Chess, A. Stochastic yet biased expression of multiple Dscam splice variants by individual cells. Nature Genet. 36, 240-246 (2004).

15. Hattori, D. et al. Robust discrimination between self and non-self neurites requires thousands of Dscam1 isoforms. Nature 461, 644-648 (2009).

16. Yamagata, M. \& Sanes, J. R. Dscam and Sidekick proteins direct lamina-specific synaptic connections in vertebrate retina. Nature 451, 465-469 (2008).

17. Fuerst, P. G., Koizumi, A., Masland, R. H. \& Burgess, R. W. Neurite arborization and mosaic spacing in the mouse retina require DSCAM. Nature $451,470-474$ (2008).

18. Fuerst, P. G. et al. DSCAM and DSCAML1 function in self-avoidance in multiple cell types in the developing mouse retina. Neuron 64, 484-497 (2009)

19. Sanes, J. R. \& Zipursky, S. L. Design principles of insect and vertebrate visual systems. Neuron 66, 15-36 (2010).

20. Wang, X. et al. Gamma protocadherins are required for survival of spinal interneurons. Neuron 36, 843-854 (2002).

21. Prasad, T., Wang, X., Gray, P. A. \& Weiner, J. A. A differential developmental pattern of spinal interneuron apoptosis during synaptogenesis: insights from genetic analyses of the protocadherin- $\gamma$ gene cluster. Development 135, 4153-4164 (2008).

22. Lefebvre, J. L., Zhang, Y., Meister, M., Wang, X. \& Sanes, J. R. $\gamma$-Protocadherins regulate neuronal survival but are dispensable for circuit formation in retina. Development 135, 4141-4151 (2008).

23. Weiner, J. A., Wang, X., Tapia, J. C. \& Sanes, J. R. Gamma protocadherins are required for synaptic development in the spinal cord. Proc. NatlAcad. Sci. USA 102, 8-14 (2005).

24. Stacy, R. C. \& Wong, R. O. Developmental relationship between cholinergic amacrine cell processes and ganglion cell dendrites of the mouse retina. J. Comp. Neurol. 456, 154-166 (2003).

25. Lee, S. \& Zhou, Z. J. The synaptic mechanism of direction selectivity in distal processes of starburst amacrine cells. Neuron 51, 787-799 (2006).

26. White, F. A., Keller-Peck, C. R., Knudson, C. M., Korsmeyer, S. J. \& Snider, W. D. Widespread elimination of naturally occurring neuronal death in Bax-deficient mice. J. Neurosci. 18, 1428-1439 (1998).

27. Jelinek, H. F. \& Fernandez, E. Neurons and fractals: how reliable and useful are calculations of fractal dimensions? J. Neurosci. Methods 81, 9-18 (1998).

28. Chen, W. V. et al. Functional significance of isoform diversification in the protocadherin gamma gene cluster. Neuron. (in the press).

29. Wang, J. et al. Transmembrane/juxtamembrane domain-dependent Dscam distribution and function during mushroom body neuronal morphogenesis. Neuron 43, 663-672 (2004).

30. Kaneko, M. et al. Remodeling of monoplanar Purkinje cell dendrites during cerebellar circuit formation. PLoS ONE 6, e20108 (2011)

Supplementary Information is linked to the online version of the paper at www.nature.com/nature.

Acknowledgements We thank members of our laboratory for providing advice and reagents, including D. Cai and K. Cohen (rAAV), I.-J. Kim (fst/4-line 1 mice) and M. Yamagata for modified Rosa-CAG targeting vector. We also thank B. Stevens (Children's Hospital) for advice on culture methods. This work was supported by grants from NIH to J.R.S. (R01NS029169 and R01EY022073) and T.M. (R01NS043915) and NARSAD Young Investigator Award to J.L.L.

Author Contributions J.L.L., D.K. and J.R.S. designed experiments and prepared the manuscript. J.L.L. and D.K. performed experiments and data analysis. J.R.S. supervised the project. W.V.C. and T.M. generated $P c d h g^{\text {tako }}$ and $P c d h{ }^{\text {tcko }}{ }^{\text {mice. All authors }}$ commented on the manuscript.

Author Information Reprints and permissions information is available at www.nature.com/reprints. The authors declare no competing financial interests. Readers are welcome to comment on the online version of this article at www.nature.com/nature. Correspondence and requests for materials should be addressed to J.R.S. (sanesj@mcb.harvard.edu). 


\section{METHODS}

Mouse strains. The Pcdhg ${ }^{f c o n} 3$ conditional mutant allele, in which the third constant exon is flanked by loxP sequences and which generates a functionally null allele following Cre recombination, was described previously ${ }^{21,22}$. Retinaspecific Chx10-cre $e^{31}$ and Six3-cre transgenic mice ${ }^{32}$ were provided by C. Cepko (Harvard) and W. Klein (M.D. Anderson Cancer Center), respectively. Bax ${ }^{-/-}$ mutants $^{33}$, Purkinje-specific L7Bac-cre transgenic mice ${ }^{34}$, Chat-cre, in which the Cre recombinase gene was targeted to the endogenous ChAT gene ${ }^{35}$, and RosaCAG-LoxP-STOP-LoxP-tdTomato-WPRE reporter mice ${ }^{36}$ were obtained from Jackson Laboratories. A line of BAC transgenic mice in which regulatory elements from the $f_{s t l} t 4$ gene drive expression of CreER was generated as described in ref. 37. In this line, called line 1 to distinguish it from the line called 'BD' in ref. 37, CreER was expressed in SACs, as well as sparse other amacrine cells. We believe that expression reflects influences at the site of transgene integration rather than expression of $f_{s t l}$. Thy1-OFP3 transgenic mice, in which Thy 1 promoter and regulatory elements direct expression of Kusabira Orange (OFP) in SACs and subsets of retinal ganglion cells (RGCs), were described previously ${ }^{37}$. Pcdh ${ }^{t c k o}$ and Pcdhg ${ }^{\text {tako }}$ mice were generated using standard gene targeting techniques ${ }^{28}$. Mice were maintained on a C57/B6J background. All experiments were carried out in accordance with protocols approved by the Harvard University Standing Committee on the Use of Animals in Research and Teaching.

Generation of single Pcdhg isoform conditional knock-in mice. Pcdhgal and $p c d h g c 3$ full-length cDNAs were amplified from RNA isolated from P21 C57/BL6 mouse brain, and cloned in frame into pCMV-mCherry-N1 (Clontech). Linker sequence residing between the third constant exon and $g f p$ in Pcdhg ${ }^{\text {fusg }}$ knock-in mice and shown to produce functional Pcdhg-GFP fusion proteins in vivo ${ }^{20}$ was subcloned into pCMV-pcdhgal/c3-mCherry-N1. Targeting vector pRosa26$\mathrm{PAS}^{38}$ was modified as described in ref. 39 to include a CAG cassette (chicken $\beta$-actin promoter and CMV immediate-early enhancer), a Gateway RfA destination site (Invitrogen), a WPRE fragment (woodchuck hepatitis virus posttranscriptional element), and a STOP sequence was cloned from pBS302 (Addgene plasmid 11925). LoxP-STOP-loxP-Pcdhgal/c3-mCherry was recombined into pROSA26CAG-Rfa-WPRE-FNF-iSceI, creating pROSA26-CAG-loxP-STOP-loxPPcdhga1/c3-mCherry-WPRE-FNF-iSceI targeting vectors. The iSceI-linearized vectors were electroporated into 129/B6 F1 hybrid ES cell line V6.5. G418resistant, targeted ES clones were identified by PCR: $1.7 \mathrm{~kb}$ fragment amplified by 5'-Rosa-F: GGCGGACTGGCGGGACTA and 5'-CAG-R: CCAGGCGGGCC ATTTACCGTAAG; and $8.2 \mathrm{~kb}$ fragment amplified by $3^{\prime}$-CherryF: CTCCCA CAACGAGGACTACACCATC and 3'-RosaR: GCATTTTAAAAGCATGAAAC TACAAC. ES cell transfections and blastocyst injections were performed by the Genome Modification Facility, Harvard University. Following germ-line transmission, the FRT-neo-FRT cassette was excised by crossing to mice that express Flp recombinase ubiquitously ${ }^{40}$. Gt(ROSA)26Sor::CAG-loxP-STOP-loxP-Pcdhga1/ $c 3-m$ Cherry conditional knock-in mice are called $c A 1-m$ Cherry and $c C 3-m C h e r r y$. Labelling of neurons. Plasmid encoding pAAV2/2-CAG-palmitoylation tagmCherry-WPRE was used to generate recombinant AAV2/2 expressing membrane-tagged Cherry. To label SACs in retina expressing $c C 3$ - $m$ Cherry or cA1-mCherry, we used rAAV2/2-CBA-YC3.6-WPRE expressing a calcium sensor that includes cytosolic YFP and used here for visualization of neuronal morphology ${ }^{41}$. Recombinant AAV2/2-CAG-memb-mCherry and rAAV2/2YC3.6 were prepared at the Harvard Gene Therapy Institute $\left((1-2) \times 10^{12}\right.$ genome copies per $\mathrm{ml})$. Optimal titres of $(1-2) \times 10^{9}$ viral genome particles per $\mathrm{ml}$ for AAV2/2-CAG-memb-mCherry and $2 \times 10^{10}$ viral genome particles per $\mathrm{ml}$ for rAAV2/2- YC3.6 were prepared in phosphate-buffered saline (PBS, $\mathrm{pH}=7.4)$. rAAV2/9 expressing GFP and mCherry were generated and provided by D. Cai and K. Cohen in our laboratory; high titre virus was produced at the University of Pennsylvania Vector Core.

To inject virus into eyes, adult mice were anaesthetized with ketamine/xylazine by intraperitoneal injection. A $301 / 2 \mathrm{G}$ needle was used to make a small hole in the temporal eye, below the cornea, and $1.5 \mu \mathrm{l}$ of rAAV virus was injected into the vitreous humour with a Hamilton syringe and $33 \mathrm{G}$ blunt-ended needle. Animals were killed and retinas were dissected 4-6 weeks following injection. For cerebellar virus infection, P1-P2 mice were anaesthetized with ice and a small puncture was made into the caudal-medial position of one cortical lobe; $1.5 \mu \mathrm{l}$ of $\mathrm{rAAV} 2 / 9-\mathrm{GFP}$; mCherry virus was injected with a Hamilton syringe and $33 \mathrm{G}$ blunt-ended needle. Mice were analysed 12-35 days after infection.

For biolistic transfection of SACs, gold particles $(1.0 \mu \mathrm{m}$ diameter, Bio-Rad) were coated with plasmids encoding tdTomato driven by CMV promoter ${ }^{24}$. Live retinas were dissected, transected with four radial incisions, flattened with photoreceptor side down, and mounted onto a nitrocellulose filter (Millipore). Gold particles were delivered using a Biolistics Helios Gene gun device (Bio-Rad), and retinas were cultured in Ames medium (Sigma) in an oxygenated incubator heated to $37^{\circ} \mathrm{C}$ for $12-16 \mathrm{~h}$.
To assess interactions between dendrites of neighbouring SACs, we injected pairs of cells with fluorescent dyes. Retinas from mice expressing OFP in SACs (Thy1-OFP3) were mounted RGC side up and perfused with Ames medium bubbled with $95 \% \mathrm{O}_{2} / 5 \% \mathrm{CO}_{2}$ at $25^{\circ} \mathrm{C}$. OFP ${ }^{+}$SACs were visualized with epifluorescence, and impaled with high resistance electrodes $(50 \mathrm{M} \Omega)$ filled with a $\mathrm{K}^{+}$ based intracellular recording solution supplemented with $50 \mu \mathrm{M}$ Alexa Fluor 568 (for targeting) and $200 \mu \mathrm{M}$ of Alexa Fluor 488 or 647 (for filling, Invitrogen). Square voltage pulses of $\sim 3 \mathrm{~V}$ were applied to SACs at $50 \mathrm{~Hz}$ using a BK Precision Model 3011B function generator. After filling one SAC, the electrode was replaced with a second containing the contrasting dye and the second cell was filled. Images of labelled SAC pairs in live retinas were acquired at $40 \times$ on a Zeiss LSM 510 confocal microscope.

Tissue preparation and immunohistochemistry. Mice were killed with intraperitoneal injection of Nembutal, and either enucleated immediately or transcardially perfused with Ringer's solution followed by $4 \%$ paraformaldehyde (PFA) in PBS Eye cups were removed and fixed in $4 \%$ PFA on ice for $1 \mathrm{~h}$, followed by dissection and post-fixation of retinas for an additional $30 \mathrm{~min}$, then rinsed with PBS. Brains were post-fixed in $4 \% \mathrm{PFA}$ at $4{ }^{\circ} \mathrm{C}$ overnight. Animal procedures were in compliance with the US National Institutes of Health Guide for the Care and Use of Laboratory Animals and approved by the Animal and Care and Use Program at Harvard University.

Whole-mount preparations and cryosections of retinas were performed as described $^{22,42}$. Briefly, whole retinas were incubated for $1-2 \mathrm{~h}$ in blocking buffer ( $0.4 \%$ Triton-X, $4 \%$ normal donkey serum in PBS), then incubated for 6 days at $4{ }^{\circ} \mathrm{C}$ with primary antibodies. Sagittal $80 \mu \mathrm{m}$ sections of cerebellum were obtained with a vibratome (Leica), incubated in blocking buffer, and with primary antibodies for 2 days at $4{ }^{\circ} \mathrm{C}$. Following washing, retinas and brain sections were incubated for $3 \mathrm{~h}$ at room temperature with Alexa-conjugated secondary antibodies (Invitrogen or Jackson ImmunoResearch). Whole retinas were flattened with photoreceptor side down onto nitrocellulose filters. Retina flat-mounts and brain sections were mounted onto glass slides, covered with Vectashield (Vector) or Fluoromount G (Southern Biotech), and imaged on an Olympus FV1000 scanning confocal microscope. Antibodies used were as follows: chick and rabbit anti-GFP (Aves and Millipore); rabbit anti-DsRed (Clontech); goat anti-choline acetyltransferase (Millipore); guinea pig anti-vGluT3 (Millipore); rabbit anti-Calbindin (Swant); mouse anti-syntaxin HPC1 clone (Sigma); rabbit anti-cleaved caspase3 (Cell Signaling Technology). Nuclei were labelled with DAPI, Po-pro1, or NeuroTrace Nissl 435/455 (Invitrogen).

SAC purification and culture. To isolate and culture wild-type and $P c d h g$ mutant SACs in vitro, we crossed the Thy1-OFP3 transgene, which selectively directs expression of Kusabira Orange (OFP) in SACs and subset of $\mathrm{RGCs}^{37}$, into Pcdhg $g^{f c o n 3}$; Six3-cre mice. Retinas from genotyped Pcdhg fcon3/fcon3; Six3-cre; Thy1-OFP3 mutant and control P2 mice were dissociated using papain ${ }^{22}$. $\mathrm{OFP}^{+}$ SACs were isolated by fluorescence activated cell sorting (FACS, MoFlo), plated onto poly-L-lysine-coated glass coverslips (Warner) and cultured for 7-9 days in RGC growth media modified from Meyer-Franke ${ }^{43}$ in the following ways: (1) substitution of NS21 ${ }^{44}$ for B27, (2) substitution of N2 (Invitrogen) for Sato stock, (3) addition of TGF- $\beta 1$ and TGF- $\beta 2$ ( $2.5 \mathrm{ng} \mathrm{ml}^{-1}$; Peprotech), and (4) addition of mouse glia-conditioned medium (15\%). One-third of media was exchanged with fresh media every three days. Cells were fixed with cold $4 \% \mathrm{PFA} / 4 \%$ sucrose for $15 \mathrm{~min}$, and immunostained for syntaxin and calbindin to confirm SAC identity, and for GFP to confirm $P c d h g^{-/-}$; GFP ${ }^{-}$SACs from unrecombined Pcdhg-GFP ${ }^{+}$ SACs due to variegated Six3-Cre activity in retina.

Image analysis. For best reproduction and clarity of SAC arborizations, maximized projections of confocal images were inverted and contrast-enhanced using Photoshop (Adobe Systems). For morphometric analysis of SACs, we used Fiji software and selected confocal image series of wild-type and Pcdhg mutant SACs situated in comparable retinal eccentricities. Self-crossings per dendritic branch order were quantified as number of branch overlaps detected in single confocal planes; crossings occurring distal to fifth branch order could not be quantified accurately owing to severity of defects in mutants. Dendritic field diameter was measured as the longest axis of arborization. In some cases, arborizations were re-imaged by oversampling using a $60 \times 1.45 \mathrm{NA}$ objective at $x, y, z$ resolution of $47 \times 47 \times 131 \mu \mathrm{m}$ and then subjected to deconvolution using Huygens software (http://www.svi.nl/HuygensProfessional).

For analysis of SAC density and mosaic regularity, confocal $z$-stacks of ChATlabelled SACs through the GCL and INL were acquired at similar locations in central retina. Sample sizes were $4-5$ areas $\left(0.099 \mathrm{~mm}^{2}\right)$ per animal, 2-4 animals per genotype. For each field, $x-y$ coordinates of SAC arrays were obtained by manually marking centres of cells using Fiji and used to compute SAC density (number per $\mathrm{mm}^{2}$ ), packing factor ${ }^{45}$, and density recovery profiles (DRP) ${ }^{46}$ with WinDRP software (http://www.mpimf-heidelberg.mpg.de/ teuler/WinDRP/ ReadMe.htm). 
To compare the space-filling and complexity of control and mutant SAC arborizations, we computed fractal dimensions, $D f$, which provide a measure of how completely dendrites fill its area $a^{2,27,47,48}$. To calculate $D_{\mathrm{f}}$, we applied the boxcounting method as implemented in the FracLac 2.5 plug-in for ImageJ software (http://rsb.info.nih.gov/ij/plugins/fraclac/FLHelp/Introduction.htm; NIH). Confocal images of cultured mutant and control SACs were obtained at equivalent laser scanning parameters with a $60 \times$ oil immersion lens, and maximum projections and thresholded, binary images were processed using Image J. Box counts using a series of progressively smaller box sizes $(d)$ were scanned in a region of interest covering the SAC arborization, and the number of boxes intersected by pixels $[k(d)]$ were analysed; this computes $D_{\mathrm{f}}$, which represents an inverse linear regression between $\log [k(d)]$ and $\log (d) . D_{\mathrm{f}}$ ranges from 1.0 (straight line with a dimension of 1) to 2.0 (plane with a dimension of 2); a difference of 0.1 represents a doubling of complexity ${ }^{27}$.

For analysis of dendrite overlap between arborizations of neighbouring SACs, pairs with somata separated by $80-160 \mu \mathrm{m}$ were selected because their dendrites are known to interact ${ }^{25}$. Images were processed using Fiji or Photoshop software. To estimate the amount of dendritic overlap that would occur by chance if two SAC arborizations occupy the same territory, we flipped or rotated the image of one SAC, realigned cell body position, and merged images. This method was inspired by work on tiling of RGC dendrites ${ }^{49}$. We measured total overlapping pixels in real and flipped images, interpreting ratios of $>1$ (real/flipped) as indicating non-random interactions between SACs.

Purkinje cell dendrite self-crossings detected in single confocal planes were counted in a $7,225 \mu \mathrm{m}^{2}$ region of interest assigned to middle of arborization. Purkinje arborization areas were measured using the convex-hull selection in Fiji. Calbindin-labelled Purkinje somata residing along a $635 \mu \mathrm{m}$ segment in lobules III-VI in single confocal planes were counted to measure Purkinje cell density.

Means were compared using the two-tailed Student's $t$ test on condition of equivalent variances determined by F-test, or with the Mann-Whitney nonparametric test. Means of multiple samples were compared using ANOVA and posthoc Tukey test.

RT-PCR of dissociated retina cells. We used FACS to sort live cells from dissociated P7 whole retina, $\mathrm{VC} 1.1^{+}$amacrine cells, and $\mathrm{OFP}^{+}$;Thy $1.2^{-}$SACs cells, as described previously $y^{37,50}$. Amacrine cells were sorted from a live cell suspension of dissociated retinal cells using monoclonal VC1.1 antibody $\left(200 \mu \mathrm{g} \mathrm{ml}^{-1}\right.$, Sigma) and an anti-IgM secondary conjugated to phycoerythrin-Cy7 (Southern). OFP ${ }^{+}$ SACs were sorted from $\mathrm{OFP}^{+}$RGCs by negative selection of Thy1.2-PE-Cy7 labelled RGCs. In each condition, 2,000 cells were sorted directly into RNA lysis buffer (Qiagen); RNA was purified and first strand cDNAs were generated with Superscript RT III (Invitrogen). Primers that uniquely detect the 22 Pcdhg variable exon-constant exon spliced transcripts were adapted from ref. 21, with modifications to avoid cross-hybridization. These primers, and others used to assess purity of the sorted population, are listed in Supplementary Table 1. PCR program used is: $94^{\circ} \mathrm{C}$ for $2 \mathrm{~min} ; 30$ cycles of $94^{\circ} \mathrm{C}$ for $20 \mathrm{~s}, 56^{\circ} \mathrm{C}$ for $30 \mathrm{~s}, 72^{\circ} \mathrm{C}$ for $1 \mathrm{~min} ; 72^{\circ} \mathrm{C}$ for $7 \mathrm{~min}$.

31. Rowan, S. \& Cepko, C. L. Genetic analysis of the homeodomain transcription factor Chx10 in the retina using a novel multifunctional BAC transgenic mouse reporter Dev. Biol. 271, 388-402 (2004).

32. Furuta, Y., Lagutin, O., Hogan, B. L. \& Oliver, G. C. Retina- and ventral forebrainspecific Cre recombinase activity in transgenic mice. Genesis 26, 130-132 (2000).

33. Knudson, C. M., Tung, K. S., Tourtellotte, W. G., Brown, G. A. \& Korsmeyer, S. J. Baxdeficient mice with lymphoid hyperplasia and male germ cell death. Science $\mathbf{2 7 0}$ 96-99 (1995).

34. Zhang. X. M. et al. Highly restricted expression of Cre recombinase in cerebellar Purkinje cells. Genesis 40, 45-51 (2004).

35. Rossi, J. et al. Melanocortin- 4 receptors expressed by cholinergic neurons regulate energy balance and glucose homeostasis. Cell Metab. 13, 195-204 (2011).

36. Madisen, L. et al. A robust and high-throughput Cre reporting and characterization system for the whole mouse brain. Nature Neurosci. 13, 133-140 (2010).

37. Kay, J. N. et al. Retinal ganglion cells with distinct directional preferences differ in molecular identity, structure, and central projections. J. Neurosci. 31, 7753-7762 (2011).

38. Srinivas, S. et al. Expression of green fluorescent protein in the ureteric bud of transgenic mice: a new tool for the analysis of ureteric bud morphogenesis. Dev. Genet. 24, 241-251 (1999).

39. Yamagata, M\&. Sanes, J. R. Transgenic strategy for identifying synaptic connections in mice by fluorescence complementation (GRASP). Front. Mol. Neurosci. 5, 18 (2012).

40. Farley, F. W., Soriano, P., Steffen, L. S. \& Dymecki, S. M. Widespread recombinase expression using FLPeR (flipper) mice. Genesis 28, 106-110 (2000).

41. Kuchibhotla, K. V. et al. A $\beta$ plaques lead to aberrant regulation of calcium homeostasis in vivo resulting in structural and functional disruption of neuronal networks. Neuron 59, 214-225 (2008).

42. Hong, Y. K., Kim, I. J. \& Sanes, J. R. Stereotyped axonal arbors of retinal ganglion cell subsets in the mouse superior colliculus. J. Comp. Neurol. 519, 1691-1711 (2011).

43. Meyer-Franke, A., Kaplan, M. R., Pfrieger, F. W. \& Barres, B. A. Characterization of the signaling interactions that promote the survival and growth of developing retinal ganglion cells in culture. Neuron 15, 805-819 (1995).

44. Chen, Y. et al. NS21: re-defined and modified supplement B27 for neuronal cultures. J. Neurosci. Methods 171, 239-247 (2008).

45. Whitney, I. E., Keeley, P. W., Raven, M. A. \& Reese, B. E. Spatial patterning of cholinergic amacrine cells in the mouse retina. J. Comp. Neurol. 508, 1-12 (2008).

46. Rodieck, R. W. The density recovery profile: a method for the analysis of points in the plane applicable to retinal studies. Vis. Neurosci. 6, 95-111 (1991).

47. Montague, P. R. \& Friedlander, M. J. Morphogenesis and territorial coverage by isolated mammalian retinal ganglion cells. J. Neurosci. 11, 1440-1457 (1991)

48. Smith, T. G. Jr, Lange, G. D. \& Marks, W. B. Fractal methods and results in cellular morphology-dimensions, lacunarity and multifractals. J. Neurosci. Methods 69, 123-136 (1996).

49. Wässle, H., Peichl, L. \& Boycott, B. B. Dendritic territories of cat retinal ganglion cells. Nature 292, 344-345 (1981).

50. Kay, J. N., Voinescu, P. E., Chu, M. W. \& Sanes, J. R. Neurod6 expression defines new retinal amacrine cell subtypes and regulates their fate. Nature Neurosci. 14, 965-972 (2011). 